\title{
Long Delay Free Recall (CVLT-II)
}

National Cancer Institute

\section{Source}

National Cancer Institute. Long Delay Free Recall (CVLT-II). NCI Thesaurus. Code C120548.

The fourth part of the California Verbal Learning Test - Second Edition. After a 20-minute non-verbal task, the subject is asked to recall as many words as he or she can from List A. 